\title{
Clinical presentation and treatment of gastric metastasis from other malignancies of solid organs
}

\author{
TSUTOMU NAMIKAWA $^{1}$, ERI MUNEKAGE ${ }^{1}$, MAHO OGAWA $^{1}$, TOYOKAZU OKI ${ }^{1}$, \\ MASAYA MUNEKAGE ${ }^{1}$, HIROMICHI MAEDA ${ }^{2}$, HIROYUKI KITAGAWA ${ }^{1}$, TAKEKI SUGIMOTO ${ }^{1}$, \\ MICHIYA KOBAYASHI $^{2,3}$ and KAZUHIRO HANAZAKI ${ }^{1}$ \\ ${ }^{1}$ Department of Surgery; ${ }^{2}$ Cancer Treatment Center; ${ }^{3}$ Department of Human Health and \\ Medical Sciences, Kochi Medical School, Nankoku, Kochi 783-8505, Japan
}

Received June 30, 2017; Accepted July 5, 2017

DOI: $10.3892 /$ br.2017.943

\begin{abstract}
The aim of the present study was to analyze the clinicopathologic features and treatment outcomes of gastric metastasis from other malignancies of solid organs. A review was conducted of patients with metastatic tumors in the stomach from other malignancies of solid organs detected endoscopically at the Department of Surgery, Kochi Medical School, from January 1991 to December 2015. Seven patients (four men and three women), with a median age of 64 years (range, 42-71 years), had metastatic gastric tumors. Median tumor size was $7.3 \mathrm{~cm}$ (range, 2.5-12.0 $\mathrm{cm}$ ). The primary malignancy leading to metastatic tumors in the stomach was esophageal cancer in three patients, breast cancer in two patients, renal cell carcinoma in one patient, and ovarian cancer in one patient. Gastric metastasis presented as solitary lesions in six patients and as multiple lesions in one patient. Four patients had solitary gastric metastasis, whereas three had multiple metastases in other organs. The median tumor size was significantly smaller in patients with solitary rather than multiple metastases (4.6 vs. $12.0 \mathrm{~cm}$, respectively; $\mathrm{P}=0.038$ ). Three patients received systemic therapy and four underwent surgical resection of the metastatic tumor, and of these, only one was alive 58 months after surgery. Clinicians should be aware of the possible existence of metastatic gastric cancer, especially in breast carcinoma and esophageal cancer. Surgical resection may considerably improve patients' quality of life, and could be of benefit when there is a risk of bleeding and/or a solitary metastasis.
\end{abstract}

Correspondence to: Dr Tsutomu Namikawa, Department of Surgery, Kochi Medical School, 185-1 Kohasu, Oko-cho, Nankoku, Kochi 783-8505, Japan

E-mail: tsutomun@kochi-u.ac.jp

Key words: metastatic gastric tumor, gastric cancer, gastric metastasis, solitary metastasis

\section{Introduction}

Gastric metastases are rare, with a reported incidence of $0.2-0.7 \%$ based on clinical and autopsy findings, whereas primary gastric cancer is the most commonly diagnosed cancer worldwide and is the leading cause of cancer-related deaths (1-3). It may be very difficult to distinguish metastatic gastric tumors from primary gastric cancers based on clinical, endoscopic, radiological, and histopathological features. With gradual improvements in prognosis for cancer patients, it seems that metastatic tumors in the stomach are being encountered more frequently (1). Therefore, it is important to distinguish metastatic gastric tumor from primary gastric cancer for accurate diagnosis and optimal treatment.

Although metastatic gastric tumors are not that common, recognizing the range of possible presentations is important for the early and accurate diagnosis and treatment. The aim of the present study was to analyze the clinicopathologic features and treatment outcomes of gastric metastasis from other malignancies of solid organs.

\section{Materials and methods}

Patients and methods. Patients with metastatic tumors in the stomach from other malignancies of solid organs detected endoscopically at the Department of Surgery, Kochi Medical School, between January 1991 and December 2015 were reviewed. Diagnoses of metastatic gastric cancer were made by esophagogastroduodenoscopy (EGD), analysis of biopsy specimens, computed tomography (CT), magnetic resonance imaging, ultrasonography of the abdomen, and positron emission tomography. Patients with malignant lymphoma involving the stomach or with direct invasion from neighboring organs were excluded from the study. All the tumors were subjected to detailed examinations, including CT, ultrasonography, EGD, and pathological confirmation using biopsy or surgically resected specimens.

Statistical analysis. Correlations among continuous variables in each group were evaluated using the Mann-Whitney U-test, whereas categorical variables were evaluated using Pearson's Chi-square test. $\mathrm{P}<0.05$ was considered to indicate 
a statistically significant difference. Statistical analyses were performed using SPSS for Windows v13.0 (SPSS Inc., Chicago, IL, USA).

\section{Results}

Patient characteristics. Seven patients who had been treated for metastatic gastric tumors arising from other malignancies of solid organs were included in the present study. The clinical features of these seven patients are listed in Table I. Four patients $(57.1 \%)$ were men and three $(42.9 \%)$ were women, with patient age ranging from 42 to 71 years (median, 65 years). Four patients had lesions in the upper third of the stomach, one had lesions in the middle third of the stomach, and two had lesions in the entire stomach. Median tumor size was $7.3 \mathrm{~cm}$ (range, 2.5-12.0 cm). The primary malignancy leading to metastatic tumors in the stomach was esophageal cancer in three patients, breast cancer in two patients, renal cell carcinoma in one patient, and ovarian cancer in one patient. The pathology of both tumors arising from breast cancer showed invasive lobular carcinoma, and the pathology of the esophageal cancers showed squamous cell carcinoma. Fig. 1 shows the EGD results in a 71-year-old patient who was diagnosed with metastatic gastric cancer arising from esophageal cancer.

Gastric metastasis presented as solitary lesions in four patients and as multiple lesions in three patients. The median tumor size was significantly smaller in patients with solitary rather than multiple metastases $(4.6$ vs. $12.0 \mathrm{~cm}$, respectively; $\mathrm{P}=0.038$ ). Six patients had a single lesion in the stomach and one patient had multiple metastatic tumors in the stomach, as well as metastases to the lung, bone, liver, kidney, and skin. Examination of the tumors revealed ulcerated tumors in three patients, linitis plastica lesion in two, and a protruding tumor in another two patients. In one patient, the condition was diagnosed from biopsy specimens using endoscopic ultrasound-guided fine needle aspiration (EUS-FNA) (5).

The interval between treatment of the primary tumor and diagnosis of the metastatic tumor in the stomach was 23 years in the case of solitary gastric metastasis from renal cell carcinoma and 6 years in the case of solitary gastric metastasis from ovarian cancer. In all other cases (i.e., gastric metastases from breast cancer and esophageal cancer), presentation was synchronous.

Treatment of gastric metastases. One patient with multiple metastases arising from breast cancer was treated with chemotherapy using fluorouracil; however, this patient died 5.5 months after therapy. In the case of the patient with gastric metastasis of renal cell carcinoma presenting 23 years after radical nephrectomy, 58 months have elapsed since the surgical resection of the tumor and the patient is alive without the occurrence of any new lesions. One patient with solitary gastric metastasis of ovarian cancer underwent distal gastrectomy; however, this patient developed peritoneal metastasis and died 9 months after surgery. One patient with gastric metastasis of breast cancer is currently undergoing chemotherapy with anti-estrogen therapy, and one patient with solitary gastric metastasis of esophageal cancer is undergoing chemotherapy with fluorouracil and cisplatin. One patient with solitary gastric metastasis of esophageal cancer (which

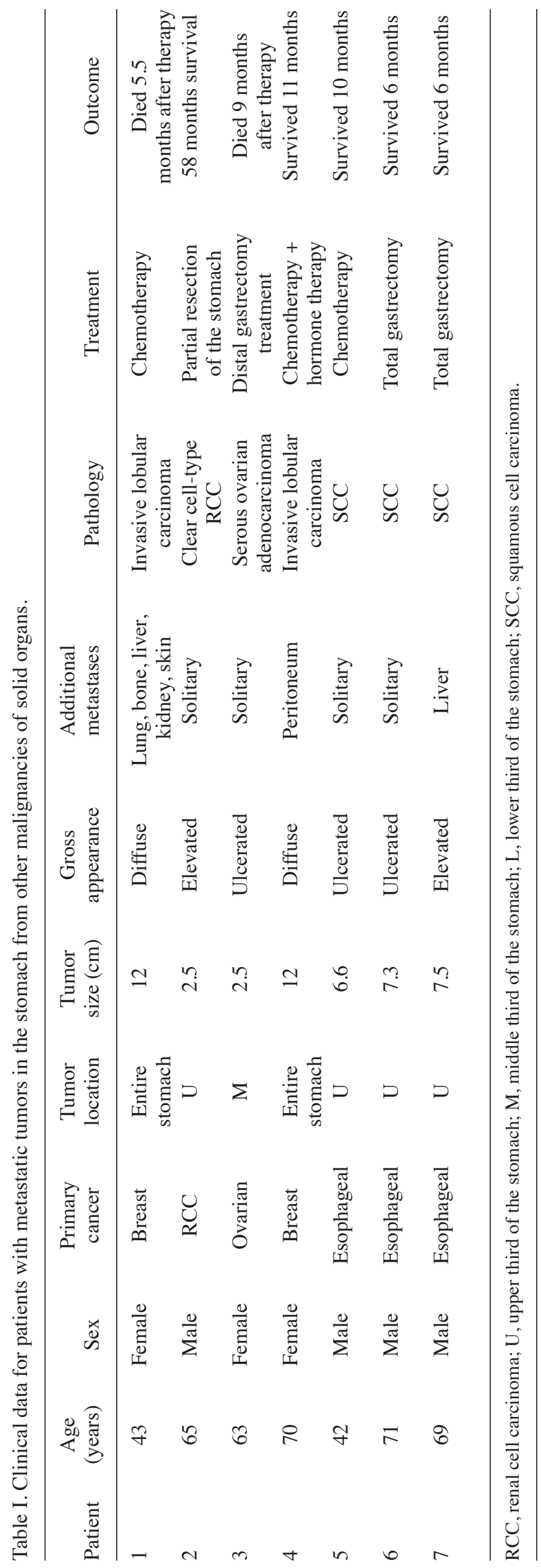




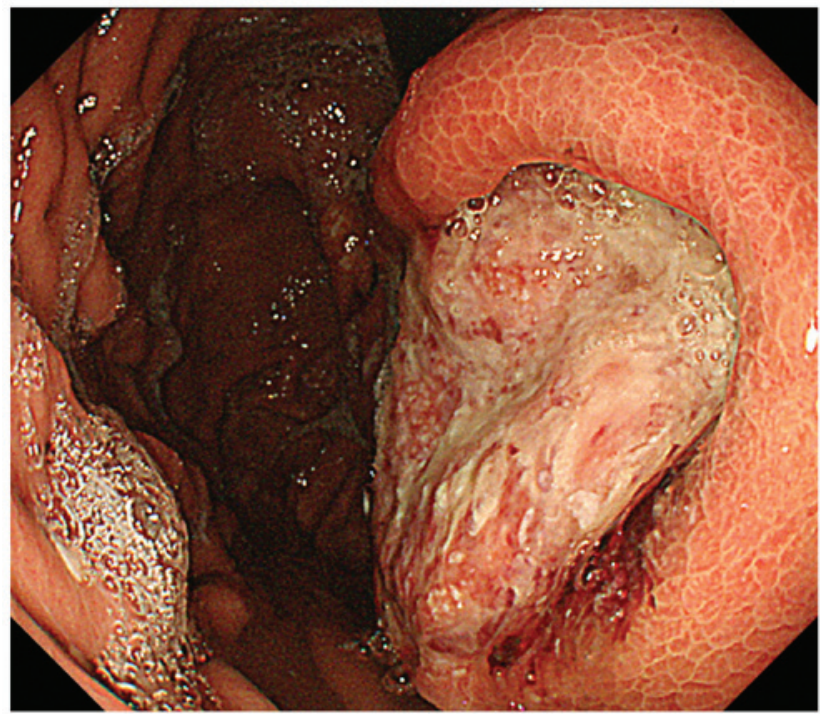

Figure 1. Endoscopic image of metastatic gastric cancer from patient 6 . Endoscopy revealed a circumscribed ulcerated lesion in the upper part of the stomach body that was diagnosed as metastatic gastric cancer arising from an esophageal squamous cancer.

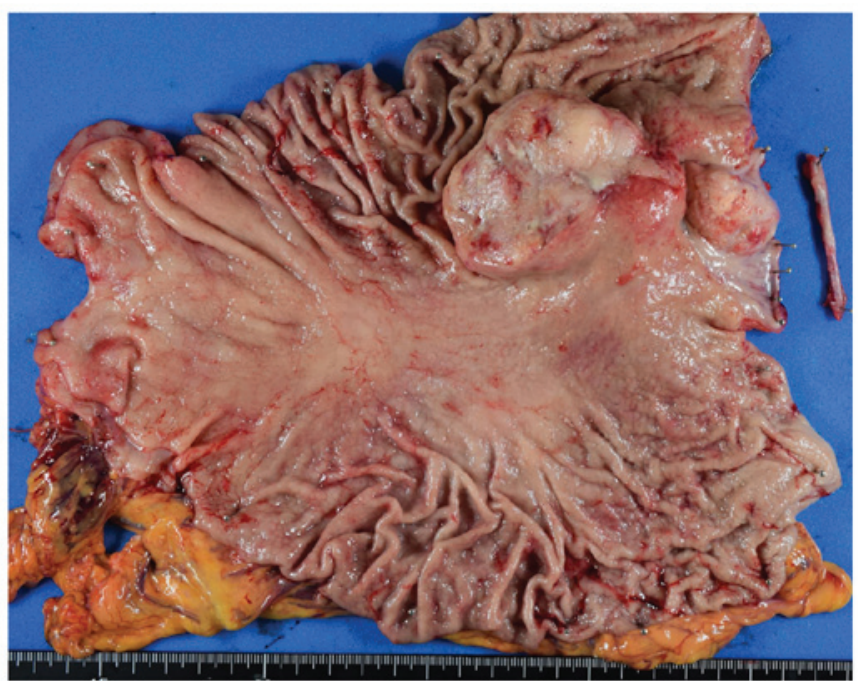

Figure 2. Gross appearance of the resected specimen from patient 6 . The lesion, a solitary gastric metastasis of esophageal cancer, was ulcerated with a distinct margin.

was an ulcerated tumor measuring $7.3 \times 7.2 \mathrm{~cm}$ ) underwent total gastrectomy and is undergoing chemotherapy with docetaxel, cisplatin, and fluorouracil post-surgery (Fig. 2). One patient with gastric and liver metastases arising from esophageal cancer is undergoing chemotherapy with docetaxel, cisplatin, and fluorouracil.

\section{Discussion}

In the present study, the incidence of esophageal and breast cancer as the primary malignancies leading to metastatic tumors in the stomach was high, which is similar to findings of previous studies reporting that the most common primary sites of metastases to the stomach are the breast, melanoma, lung, and esophagus $(1,4,5)$. Although the mechanisms underlying gastric metastasis have not been clearly elucidated, potential pathways may include peritoneal dissemination, hematogenous dissemination, lymphatic spread, and direct tumor invasion (1).

In the case of hematogenous dissemination, tumor cells may become trapped in vessels in areas of the stomach wall with a rich blood supply, such as the submucosal or subserosal layers (6). Investigations into the mechanisms responsible for gastric metastasis depending on the primary malignancy are expected in the near future. At the same time, risk factors for the development of metastatic gastric tumors need to be elucidated to enable early diagnosis and to establish the best therapeutic strategy. In addition, when planning treatment for a gastric neoplasm, it is important to differentiate a primary gastrointestinal tract tumor from a metastatic tumor, especially in patients who have previously been treated for lobular breast carcinoma or esophageal cancer.

Breast cancer is the most commonly diagnosed cancer worldwide and the second leading cause of cancer-related deaths (7). Despite breast cancer metastases to the gastrointestinal tract being less common than to other sites, because breast cancer is extremely common, it may be responsible for a high proportion of metastatic gastric tumors. In the present study, the pathological results for both breast cancer patients showed invasive lobular carcinoma, which agrees with findings reported previously $(4,8)$. As tumor histology is one of the predictors of metastatic spread, lobular carcinoma is more likely to metastasize to the gastrointestinal tract, although metastatic gastric tumors are less common than ductal carcinoma and the mechanisms involved are not clear (8-10). These results emphasize the importance of considering metastatic gastric cancer, especially in patients with a previous history of breast cancer. In such cases, clinicians should undertake additional immunohistochemical examinations, such as staining for estrogen or progesterone receptors (9).

In the present study, the metastatic gastric tumors in the two breast cancer patients exhibited a diffuse-type appearance, such as linitis plastica. One of the characteristic endoscopic features in these two cases was diffuse infiltration of the gastric wall. This finding is similar to linitis plastica, which is a diffuse-type gastric cancer that presents in the area of the fundic gland and is characterized by thickening of the stomach wall and deformation of the stomach resulting in a leather bottle-like appearance of the stomach $(1,4,8,11)$. In a previous study, the median interval to metastatic tumors in the stomach from primary breast cancer and renal cell carcinoma was 50-78 and 75.6 months, respectively (1), which highlights the fact that metastatic spread to the stomach may occur many years after initial treatment for the primary tumor.

The incidence of metastases in the stomach from esophageal carcinoma is in the range of $0-15 \%$ in autopsy cases, and $1.7 \%$ in clinical cases before autopsy (12-14). The microlymphatic system in the esophageal submucosa is thought to be continuous with that in the gastric submucosa, and this may be associated with the mechanism underlying gastric metastasis $(6,14)$. In the present study, in all three patients with gastric metastases from esophageal cancer, the metastatic tumors were located in the upper gastric body close to the esophagocardial junction, which could be explained by metastases occurring via the lymphatic system. 
Gastric metastasis arising from ovarian cancer can be diagnosed using EUS-FNA as lesions exhibiting fold convergence with a central depression. Hassan et al (15) reported that EUS-FNA significantly changed patient management in $15 \%$ of patients fit for surgery when lymph nodes or lesions were considered to be distant metastases of primary gastric cancer. In the diagnosis of metastatic gastric carcinoma, endoscopy with biopsies is the most common modality: However, results from endoscopic biopsies may be negative for tumor cells because the infiltration of tumor cells is localized to the deeper layers, which are often inaccessible to biopsy forceps. In these cases, accurate diagnosis may be supported by EUS-FNA with other radiological techniques, such as CT and positron emission tomography (16).

In the present study, the median tumor size was significantly smaller in patients with solitary rather than multiple metastases. Furthermore, in the case of solitary gastric metastasis from a renal cell carcinoma, the patient achieved long-term survival without the occurrence of any new lesions. Previous studies have also reported that patients with solitary gastric metastasis arising from renal cell carcinoma have good outcomes following treatment compared with patients with multiple metastases $(5,17)$.

One of the limitations of the present study is the small number of patients from a single institution. Thus, further studies with a larger number of patients are required to gain a better understanding of the various presentations of metastatic gastric cancer from other malignancies of solid organs.

In conclusion, clinicians should be aware of the possible existence of metastatic gastric cancer from other malignancies of solid organs, especially in breast lobular carcinoma and esophageal cancer. Although appropriate systemic treatment including chemotherapy or hormonal therapy for metastatic tumors in the stomach is the preferred treatment, surgical resection of metastatic gastric tumors may be recommended to improve patients' quality of life, when there is a risk of bleeding, tumor perforation, and/or a solitary metastasis.

\section{References}

1. Namikawa $T$ and Hanazaki K: Clinicopathological features and treatment outcomes of metastatic tumors in the stomach. Surg Today 44: 1392-1399, 2014.
2. Oda I, Kondo H, Yamao T, Saito D, Ono H, Gotoda T, Yamaguchi H, Yoshida S and Shimoda T: Metastatic tumors to the stomach: Analysis of 54 patients diagnosed at endoscopy and 347 autopsy cases. Endoscopy 33: 507-510, 2001.

3. Kobayashi O, Murakami H, Yoshida T, Cho H, Yoshikawa T, Tsuburaya A, Sairenji M, Motohashi H, Sugiyama Y and Kameda Y: Clinical diagnosis of metastatic gastric tumors: Clinicopathologic findings and prognosis of nine patients in a single cancer center. World J Surg 28: 548-551, 2004.

4. Taal BG, Peterse $\mathrm{H}$ and Boot H: Clinical presentation, endoscopic features, and treatment of gastric metastases from breast carcinoma. Cancer 89: 2214-2221, 2000.

5. Namikawa T, Munekage M, Kitagawa H, Okabayashi T, Kobayashi $\mathrm{M}$ and Hanazaki K: Metastatic gastric tumors arising from renal cell carcinoma: Clinical characteristics and outcomes of this uncommon disease. Oncol Lett 4: 631-636, 2012.

6. Hashimoto T, Arai K, Yamashita Y, Iwasaki Y and Hishima T: Characteristics of intramural metastasis in gastric cancer. Gastric Cancer 16: 537-542, 2013

7. Siegel RL, Miller KD and Jemal A: Cancer statistics, 2016. CA Cancer J Clin 66: 7-30, 2016.

8. Nazareno J, Taves D and Preiksaitis HG: Metastatic breast cancer to the gastrointestinal tract: A case series and review of the literature. World J Gastroenterol 12: 6219-6224, 2006.

9. Namikawa T, Kobayashi M and Hanazaki K: Unusual thickened gastric folds in a patient with breast cancer. Gastroenterology 152: e8-e9, 2017.

10. Borst MJ and Ingold JA: Metastatic patterns of invasive lobular versus invasive ductal carcinoma of the breast. Surgery 114: 637-641, discussion 641-642, 1993.

11. Pectasides D, Psyrri A, Pliarchopoulou K, Floros T, Papaxoinis G, Skondra M, Papatsibas G, Macheras A, Athanasas G, Arapantoni-Datioti $\mathrm{P}$, et al: Gastric metastases originating from breast cancer: Report of 8 cases and review of the literature. Anticancer Res 29: 4759-4763, 2009.

12. Anderson LL and Lad TE: Autopsy findings in squamous-cell carcinoma of the esophagus. Cancer 50: 1587-1590, 1982.

13. Mandard AM, Chasle J, Marnay J, Villedieu B, Bianco C, Roussel A, Elie $\mathrm{H}$ and Vernhes JC: Autopsy findings in 111 cases of esophageal cancer. Cancer 48: 329-335, 1981.

14. Saito T, Iizuka T, Kato H and Watanabe H: Esophageal carcinoma metastatic to the stomach. A clinicopathologic study of 35 cases. Cancer 56: 2235-2241, 1985.

15. Hassan H, Vilmann P and Sharma V: Impact of EUS-guided FNA on management of gastric carcinoma. Gastrointest Endosc 71: 500-504, 2010 .

16. Namikawa T, Kobayashi M and Hanazaki K: Metastatic gastric tumor arising from ovarian cancer. Gastrointest Endosc 79: 332-333, 2014.

17. Namikawa T, Iwabu J, Kitagawa H, Okabayashi T, Kobayashi M and Hanazaki K: Solitary gastric metastasis from a renal cell carcinoma, presenting 23 years after radical nephrectomy. Endoscopy 44 (Suppl 2 UCTN): E177-E178, 2012. 